\title{
Protection against Cancer with Medicinal Herbs via Activation of Tumor Suppressor
}

\author{
Yasuko Kitagishi, Mayumi Kobayashi, and Satoru Matsuda \\ Department of Environmental Health Science, Nara Women's University, Kita-Uoya Nishimachi, Nara 630-8506, Japan \\ Correspondence should be addressed to Satoru Matsuda, smatsuda@cc.nara-wu.ac.jp
}

Received 13 September 2012; Revised 14 October 2012; Accepted 27 October 2012

Academic Editor: Dagrun Engeset

Copyright ( $) 2012$ Yasuko Kitagishi et al. This is an open access article distributed under the Creative Commons Attribution License, which permits unrestricted use, distribution, and reproduction in any medium, provided the original work is properly cited.

Cancer remains a major cause of death, although research is ongoing for the development of more effective drugs. Some herbs have shown potential in preventing the occurrence and/or progression of cancer and other chronic diseases. They are being screened comprehensively to explore the possibility of development of feasible anticancer drugs. However, more information is required about the response to and the molecular target for specific herbs. It seems that there is a relationship between some medicinal herbs and tumor suppressor molecules which protect a cell from cancer. In this paper, we summarize the progress of recent research on herbs, with a particular focus on its anticancer role and molecular mechanisms underlying the cancer prevention property, supporting design for further research in this field.

\section{Introduction}

Many herbs have been used for a long time for demanded health benefits $[1-3]$. The medicinal herb is a plant (or a plant part) used for therapeutic properties. It is a widely recognized fact that many pharmacologically active drugs are derived from natural resources such as medicinal plants $[4,5]$. Therefore, it is reasonable to search for novel drug molecules in herbs. Actually, herb-derived compounds have provided attractive possibilities for treatment strategies. Herbal medicine products are also provided as dietary supplements that people take to improve their health. For example, St. John's wort has been used in the treatment of anxiety, stomach upset, insomnia, and so on. Historically, herbs have enjoyed a rich tradition of use both for their flavor enhancement and for their medicinal properties. While herbs present interesting possibilities for health promotion, more information is required about the physiological and pharmacological responses to and the molecular targets for specific herbs.

This paper focuses on antitumorigenic properties and anticancer contributions of herbs. Epidemiological evidences also point to herbs as dietary constituents with multiple anticancer characteristics [6]. It would be important to define appropriate strategies to achieve benefits from medicinal herbs. Studies show that some herbs can inhibit some antiapoptotic genes and activate some apoptotic genes $[7,8]$. Systematic characterization of active components in medicinal herbs and their mechanisms of action is important for providing the rationale for their efficacy. Therefore, biotechnological work has to be done in order to provide evidence for the efficacy and to bring herbs and derived compounds to clinical use. In this paper, the relationship between medicinal herbs and some tumor suppressor molecules has been reviewed with a focus on gene expression and posttranslational modifications.

\section{Herbal Medicine in Cancer Prevention}

Herbal medicine has been used since early times to treat malignancies in Asian countries [9]. The association between dietary patterns and the risk of developing breast cancer has also been shown in an Italian cohort, suggesting that a diet rich in raw vegetables and olive oil protects against breast cancer [10]. Furthermore, individuals consuming more raw vegetables, herbs, and spices have been associated with lower cancer risk [10]. Natural products are of importance in devising new drugs and providing unique ideas in cancer therapy. 
Actually, some herbs or spices have been approved to have a character of anticancer [11]. For example, the consumption of curcumin, a component of curry turmeric powder, has been reported to be a factor linked to a lower incidence of colon cancer [12]. Cells resistant to certain apoptotic inducers and/or radiation become susceptible to apoptosis when treated with curcumin. In addition, curcumin can also act as a chemopreventive agent in several cancers by suppressing colonic aberrant crypt foci formation and DNA adduct formation [12]. Furthermore, many cytotoxic chemotherapeutic agents such as etoposide are originally purified from herbs [13].

Two mechanisms have been proposed to be responsible for the anticancer action of the herbs and spices. One is via direct cytotoxic effects and the other is indirect through immunological modulatory action. The occurrence of cancer cells may be caused by the abnormal proliferation of cell or the inhibition of cellular apoptosis pathway. Many kinds of genes are involved in the cell proliferation and/or apoptotic regulation of cancer cells. The proliferation and the apoptosis of tumor cells are also affected by many factors and pathways such as drugs, radioactive ray, medicinal herbs, via the modulation of some oncogenes or tumor-suppressor genes. One of the potential anticarcinogenic mechanisms of herbs is via an immunological modulation. It is supposed that anticancer action of medicinal herbs may be attributed to its power to exercise immune potentiation, which may have various therapeutic applications in prophylaxis of opportunistic infections and malignant diseases. In recent years, an active ingredient responsible for the immunomodulation of some herbs has been found to be a form of complex polysaccharides [14]. As several plants have potential medical and biological efficacy used by patients with oncological neoplasia, further studies are necessary to evaluate those efficacies, where the efficacy is based on the molecular mechanisms.

\section{Activation and Inactivation of Tumor Suppressor Molecules}

Tumor development may be accelerated by disruption of the balance between cell proliferation and cell death, which is maintained through regulations of various signal transduction pathways. Apoptosis, also known as programmed cell death, is caused by various cell injuries including DNA damage [15]. It has been demonstrated that various cell proliferation- and apoptosis-signal transduction pathways are built on complicated networks between oncogenes and tumor suppressor genes such as p53 and its downstream factors [16]. For example, a tumor suppressor p53 controls various genetic expressions and plays an important role in cell proliferation and in modulation of signal transduction pathways. Accumulation of p53 in cells after DNA damage leads to cell cycle arrest and apoptosis induction. In addition, p53 is involved in repair of damaged DNA and, thus, prevents accumulation of mutations and suppresses tumor development [17]. Tumor suppressor molecules protect a cell from cancer. When the tumor suppressor genes lose their function, the cell may progress to cancer in combination with other genetic changes. Tumor suppressor genes regulate diverse cellular activities including DNA damage repair, cell proliferation, cell differentiation, cell migration, and programmed cell death (Figure 1). An important tumor suppressor is the p53 tumor suppressor. Other examples of tumor suppressors include Rb, PTEN, p21WAF1, p27KIP1, and APC (Figure 2) [18]. The recessive characteristics of these genes require mutations on both alleles. The p53 gene product is a transcriptional factor that blocks the progression of cell cycle. Mutations in the p53 gene are frequently found in many human cancers, and the mutation sites are localized in the conserved region of the gene [19]. Consequently, it loses its ability to bind its consensus DNA sequences, and it can neither activate the target genes nor perform its tumordefensive functions during carcinogenesis. There are two types of p53 genes: the wild-type p53 gene and the mutant p53 gene [20]. Cancerous p53 mutations usually confer the mutant protein with a dominant-negative activity over the remaining wild-type gene. Moreover, many mutant p53 forms acquire dominant-negative activities and sometimes gain oncogenic properties [21]. These activities of p53 are also regulated by posttranslational modification. Phosphorylation and acetylation state, subcellular localization, and interaction with other cellular proteins are likely to influence the function of p53 [22]. In cells facing oxidative stress and DNA-damage, for example, p53 dissociates from its ubiquitin ligase MDM2 [23], via various posttranslational modifications which promote its stabilization and activation.

$\mathrm{Rb}$ gene alteration or functional inactivation of $\mathrm{pRb}$, an $\mathrm{Rb}$ gene product, has also been reported in several kinds of cancers. The phosphorylation of the $\mathrm{pRb}$ is regulated by cyclin-dependent kinases in accordance with the cell cycle [24]. Hypophosphorylated wild-type pRb is tightly bound to the nuclear matrix and seems to be critical in the inhibition of cellular proliferation. Hyperphosphorylation is a physiological mechanism of inactivation of pRb [25]. Many cell cycle regulators modulate $\mathrm{pRb}$ function through its phosphorylation status. Active complexes of G1 cyclins and cyclin-dependent kinases inactivate $\mathrm{pRb}$ through its phosphorylation, while p21WAF1 and p27KIP1 inhibit cyclindependent kinases [26]. The $\mathrm{pRb}$ protein appears to prevent the function of transcription factors and other proteins needed for $S$ phase until its inactivation by cyclin-dependent kinases in late G1. Deregulated inactivation of pRb in G1 phase may be a universal mechanism underlying cellular transformation. As reversible protein phosphorylation plays a central role in regulating intracellular signaling, dysregulation of the mechanisms that regulate phosphorylation may play a key role in cancer initiation and maintenance. Works had focused on the role of specific protein phosphatases in malignant transformation [27].

The phenotype of the cancerous cell may arise from epigenetic events that may alter the heritable state of gene expression. Epigenetic silencing of the tumor suppressor genes is a well-established oncogenic process [28], and the reactivation of tumor suppressor genes is an attractive molecular target for cancer therapy. Epigenetic alterations, such as DNA methylation, also appear to be 


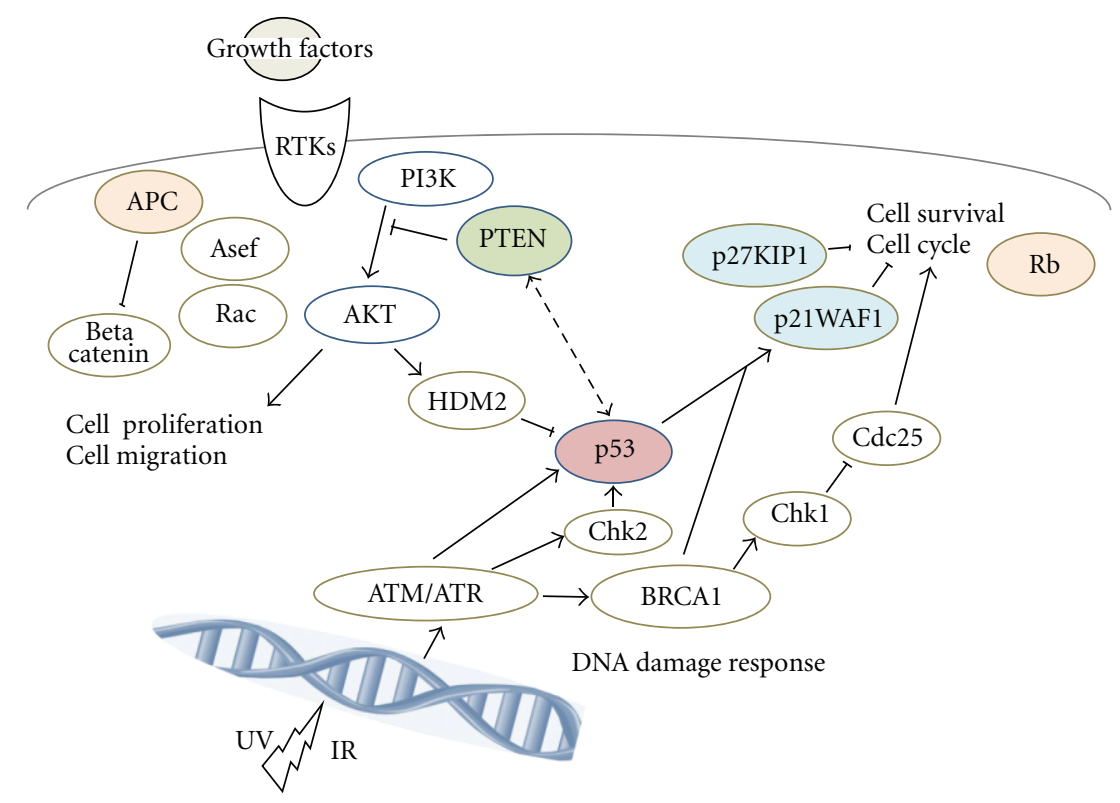

FIGURE 1: Schematic representation of tumor suppressor signaling including p53, Rb, APC, p21WAF1/p27KIP1, and PTEN. Examples of molecules known to act on cell proliferation and cell survival via the regulatory pathways are shown. Note that some critical pathways have been omitted for clarity.

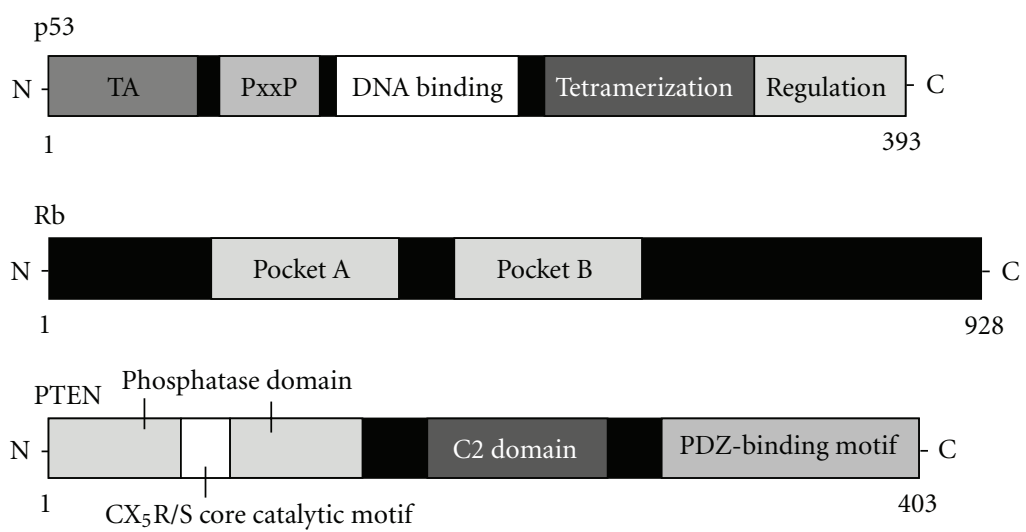

FIGURE 2: Schematic structures of p53, Rb, and PTEN proteins. The predicted consensual domain structures for each protein are depicted. The functionally important sites are also shown. Note that the sizes of protein are modified for clarity. TA: transactivation domain; PxxP: proline-rich region; pocket A, pocket B: a tandem of folds; C2 domain: a protein structural domain involved in targeting proteins to cell membranes; PDZ: a common structural domain in signaling proteins (ㅁDD95, $\underline{\text { Dlg, }} \underline{\mathrm{Z}} \mathrm{O}-1$, etc.).

tightly linked to the sequential nonreversible events of normal tissue differentiation and organogenesis [29]. Hypermethylation of $\mathrm{CpG}$ islands, an epigenetic event that is not accompanied by changes in DNA sequence, represents an alternative mechanism to inactivate tumor suppressor genes and plays a major causal role in cancer [30]. As a result of histone deacetylation and methylation, the CpG island's hypermethylation establishes a state of gene silencing. Accumulating evidence indicates that CpG island's hypermethylation is an early event in cancer development [31].

\section{Relationship between Medicinal Herbs and Tumor Suppressors}

Many herbs had been established to have a character of antitumor activity (Figure 3). Experimental studies about antitumor effects of herbs had also been found [32]. On the other hand, many kinds of tumor suppressor genes had been shown to be involved with cell proliferation and apoptotic regulation of cancer cells. There might be a substantial relationship between medicinal herbs and tumor suppressors. 


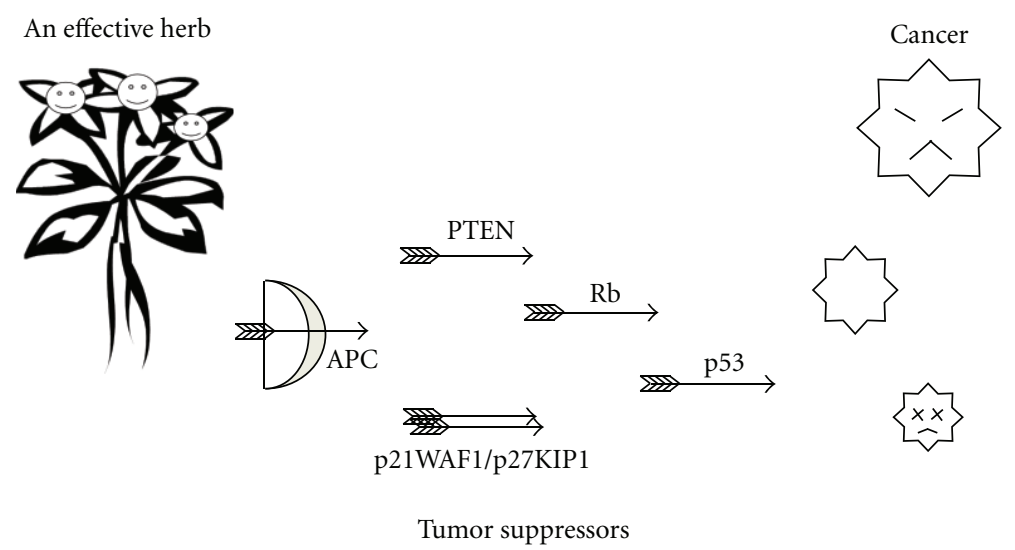

Figure 3: Tumor-suppressor-dependent anticancer function of a medicinal herb. Schematic illustrations of the tentative model for the anticancer function of herbs are shown. Herbs can stimulate tumor suppressor activities against cancer, which can also contribute to cancer prevention.

4.1. p53. Scutellaria baicalensis is used as an adjuvant to cancer chemotherapy. Following the treatment, the altered cellular protein expression indicates that cell growth arrest and apoptosis are potential mechanisms of its cytotoxicity. Increased expression of $\mathrm{p} 53$ in the cancer cells of key proteins related to the enhancement of apoptosis is observed [33]. Similarly, Gleditsia sinensis thorns are used as a medicinal herb, which shows a decrease in cell growth and an increase in cell cycle arrest during the G2/M-phase. The arrest is correlated with increased p53 levels and downregulation of cyclinB1 [34]. The mRNA level of the wild-type p53 gene also increases with the treatment of Kanglaite, an extract from Coix seed. It can be assumed that Kanglaite extends half-life of p53 protein, by which Kanglaite may induce the apoptosis of tumor cell [35]. A Ginsenoside, one of components in American ginseng herb, increases levels of Bax protein and induces cell death, activating the p53 tumor suppressor [36]. Knockout of p53 dramatically decreases the cell death, suggesting that p53 contributes to apoptosis induced by Ginsenoside in the cancer cells. Thymoquinone, the most abundant component in black seed, is a dietary chemopreventive agent against cancer. Apoptosis induction by thymoquinone is associated with an increased p53 mRNA expression and the downstream p53 target genes [37]. Treatment with a specific inhibitor of p53 restores p 21 WAF1 level to the untreated control and suppresses the cell cycle arrest and apoptosis. The apoptotic effect of thymoquinone is, thus, linked to $\mathrm{p} 53$.

4.2. $R b$. The honokiol, a component of oriental herb Magnolia officinalis, treatment decreases the viability of PC-3 and LNCaP human prostate cancer cells in a concentrationand time-dependent manner with G0-G1 phase cell cycle arrest. The honokiol-treated PC-3 and LNCaP cells exhibited a marked decrease in the levels of total retinoblastoma protein $(\mathrm{Rb})$, which correlated with the suppression of transcriptional activity of E2F1 [38]. Triptolide, a purified extract from a herb Tripterygium wilfordii hook $F$, exhibits antiproliferative and proapoptotic function. Triptolide shows increased p21(cip1) expression and reduced $\mathrm{pRb}$ phosphorylation [39]. Acanthopanax gracilistylus, a medicinal herb, markedly inhibits the proliferation of several cancer cell lines such as MT-2, Raji, HL-60, TMK-1, and HSC-2 [40]. The mechanism of the inhibition of the cell growth involves arrest of the cell cycle at the $\mathrm{G}(0) / \mathrm{G}(1)$ stage, being accompanied by a decrease of phosphorylated $\mathrm{pRb}$ protein and reduced Cdk2 and Cdk4. Licochalcone is a novel estrogenic flavonoid isolated from a herb licorice root and shows antitumor activity in various human cell lines. Licochalcone also inhibits phosphorylation of $\mathrm{pRb}$, specifically phosphorylation of S780, and reduces expression of transcription factor E2F; cyclins D1, Cdk4, and Cdk6 [41, 42]. Similarly, Dichloromethane from cape aloe extract inhibits cell proliferation, which is also associated with decreased pRb phosphorylation [43]. In addition, multiherb anti-inflammatory product Zyflamend downregulates phosphorylation of $\mathrm{pRb}$ protein [44].

4.3. PTEN. The Honokiol is demonstrated to attenuate the angiogenic activities of human endothelial cells, which can attenuate the PI3 K/Akt/mTOR (mammalian target of rapamycin) signaling by downregulation of Akt phosphorylation and upregulation of PTEN (phosphatase and Tensin homolog deleted on chromosome 10) expression $[45,46]$. Combination of honokiol with the mTOR inhibitor rapamycin presents synergistic effects on induction of apoptosis of cancer cells. Curcumin, an active ingredient derived from the rhizome of the plant Curcuma longa, has anticancer activity. PTEN enhances curcumin-induced apoptosis, whereas inactive PTEN (G129E and G129R) inhibited curcumin-induced apoptosis [47]. In vitro studies have revealed that curcumin and resveratrol synergistically inhibit cell growth and induce apoptosis [48]. Molecular targets including phosphorylated Akt, cyclinD1, mTOR, and androgen receptor are down-regulated by the combination of curcumin and resveratrol due to the activation of PTEN, suggesting that some herbs may reduce cancer incidence 
due to the PTEN function. However, some component(s) of Rosemary herb inhibits the expression of PTEN in K562 myeloid cell line cells [49].

4.4. p21WAF1 and p27KIP1. An extract of Magnolia officinalis inhibits cell proliferation in cultured urinary cancer cells. The inhibition of proliferation is associated with G1 cell cycle arrest. Treatment with $M$. officinalis extract upregulates the expressions of p21WAF1 and p27KIP1, which are CDK inhibitors [50]. The protein product of p21WAFI can combine with cell circle protein, Cdks, and proliferating cell nuclear antigen to form a complex that can depress the cell growth. M. officinalis-extract-induced cell growth inhibition also appears to be linked to the activation of p38 MAP kinase through the p21WAF1 expression [50]. Baicalin, a herbderived flavonoid compound, has been shown to induce apoptosis and growth inhibition in cancer cells through multiple pathways. Baicalin may be a novel, adjunctive therapy for malignancies including prostate cancer. Baicalin inhibits the proliferation of LNCaP and PC3 prostate cancer cell lines. Concomitantly, baicalin enhances the expression of the cyclin-dependent kinase inhibitor, p27 Kip1, in the LNCaP cells [51]. Treatment with ethanol extract of Gleditsia sinensis thorns on vascular smooth muscle cells leads to a decrease in cell growth by arresting cells in the G2/Mphase of the cell cycle, which is associated with upregulated p21WAF1 levels [52]. Conversely, the p21WAF1 expression is blocked by treatment with the p38 MAPK-specific inhibitor SB203580. While upregulating the expression of p53 protein, Kanglaite can also raise the expression of p21WAF1, suggesting that Kanglaite can induce apoptosis of cancer cell by way of the p53-dependent manner. However, treatment with ethyl acetate extract of Saussurea involucratat results in growth inhibition with G1 phase cell cycle arrest and apoptosis in PC 3 cells. The treatment also induces p21WAF1 and p27KIP1 expression, independent of the p53 pathway [53].

4.5. APC. The mRNA and protein expression level of the tumor suppressor genes adenomatous polyposis coli (APC) is increased following treatment with trichosanthin, a bioactive component isolated from herbal plant Trichosanthes kirilowii Maximowicz, in HeLa cells [54]. Methylationspecific PCR (MSP) detection showed that trichosanthin induced demethylation in the HeLa cells and that this demethylation activity was accompanied by the decreased expression of DNMT1 and reduced DNMT1 enzyme activity. Trichosanthin is capable of restoring the expression of methylation-silenced tumor suppressor genes. It is potentially useful as a de-methylation agent for the clinical treatment of cancers. Carnosol, a component of rosemary, prevents APC-associated carcinogenesis, potentially via its ability to enhance E-cadherin-mediated adhesion and suppress beta-catenin tyrosine phosphorylation [55]. Dietary administration of carnosol may decrease intestinal cancer cell growth.
4.6. Perspective. In conclusion, medicinal herbs could serve as a promising approach for cancer treatment. The information here may provide further insight into the molecular mechanisms underlying the clinical use of the herbs (e.g., ginsenoside and Kanglaite) as a cancer therapy. The identification of target molecules relevant to diseases allows screening for natural products capable of modulating these targets. This may represent the basis for the development of rational treatment of diseases such as cancer. As it is important to identify a set of genes related to the drug's sensitivity to cancer cells in order to establish a predictive method, this kind of research also opens avenues for the prediction of the response of individual cancer patients to therapy. As we have already had knowledge and experience on medicinal herbs for centuries, drug developments from the natural herb products should be differently approached from the conventional pharmaceutical pattern. Indeed, the isolation and elucidation of their chemical structure enable pharmacological and molecular biological investigations comparable to chemically synthesized compounds. It is, however, not possible to predict which individual cases of cancer patients will respond to proposed therapy. The concept of individualized tumor therapy is of importance for traditional herbal medicines. Therefore, detailed mechanisms underlying the effects of herbs should be further investigated in the near future, aiming to obtain such information for the potential of increasing cancer specificity and reducing adverse side effects on normal tissues.

\section{Conflict of Interests}

The authors declare that they have no conflict of interests.

\section{Authors' Contribution}

Y. Kitagishi and S. Matsuda contributed equally to this work.

\section{Acknowledgments}

This work was supported by Grants-in-Aid from the Ministry of Education, Culture, Sports, Science and Technology in Japan. In addition, this work was supported in part by the Grant from SHIN-EI Pharmaceutical Co., Ltd.

\section{References}

[1] N. S. Chauhan, V. Sharma, M. Thakur, and V. K. Dixit, "Curculigo orchioides: the black gold with numerous health benefits," Journal of Chinese Integrative Medicine, vol. 8, no. 7, pp. 613-623, 2010.

[2] B. H. May, A. L. Zhang, W. Zhou, C. J. Lu, S. Deng, and C. C. Xue, "Oral herbal medicines for psoriasis: a review of clinical studies," Chinese Journal of Integrative Medicine, vol. 18, no. 3 , pp. 172-178, 2012.

[3] M. S. Butt and M. T. Sultan, "Ginger and its health claims: molecular aspects," Critical Reviews in Food Science and Nutrition, vol. 51, no. 5, pp. 383-393, 2011. 
[4] A. Zong, H. Cao, and F. Wang, "Anticancer polysaccharides from natural resources: a review of recent research," Carbohydrate Polymers, vol. 90, no. 4, pp. 1395-1410, 2012.

[5] T. Efferth and E. Koch, "Complex interactions between Phytochemicals. The Multi-Target Therapeutic concept of Phytotherapy," Current Drug Targets, vol. 12, no. 1, pp. 122132, 2011.

[6] C. M. Kaefer and J. A. Milner, "The role of herbs and spices in cancer prevention," Journal of Nutritional Biochemistry, vol. 19, no. 6, pp. 347-361, 2008.

[7] H. T. Zhang, H. Luo, J. Wu et al., "Galangin induces apoptosis of hepatocellular carcinoma cells via the mitochondrial pathway," World Journal of Gastroenterology, vol. 16, no. 27, pp. 3377-3384, 2010.

[8] Y. Zhang and D. Liu, "Flavonol kaempferol improves chronic hyperglycemia-impaired pancreatic beta-cell viability and insulin secretory function," European Journal of Pharmacology, vol. 670, no. 1, pp. 325-332, 2011.

[9] T. Efferth, H. Miyachi, and H. Bartsch, "Pharmacogenomics of a traditional Japanese herbal medicine (Kampo) for cancer therapy," Cancer Genomics and Proteomics, vol. 4, no. 2, pp. 81-91, 2007.

[10] S. Sieri, V. Krogh, V. Pala et al., "Dietary patterns and risk of breast cancer in the ORDET cohort," Cancer Epidemiology Biomarkers and Prevention, vol. 13, no. 4, pp. 567-572, 2004.

[11] S. Wang, X. Wu, M. Tan et al., "Fighting fire with fire: poisonous Chinese herbal medicine for cancer therapy," Journal of Ethnopharmacology, vol. 140, no. 1, pp. 33-45, 2012.

[12] S. Singh and A. Khar, "Biological effects of curcumin and its role in cancer chemoprevention and therapy," Anti-Cancer Agents in Medicinal Chemistry, vol. 6, no. 3, pp. 259-270, 2006.

[13] N. J. Vogelzang, D. raghavan, and B. J. kennedy, "VP-16213 (etoposide): the mandrake root from issyk-kul," American Journal of Medicine, vol. 72, no. 1, pp. 136-144, 1982.

[14] X. L. Zhu, A. F. Chen, and Z. B. Lin, "Ganoderma lucidum polysaccharides enhance the function of immunological effector cells in immunosuppressed mice," Journal of Ethnopharmacology, vol. 111, no. 2, pp. 219-226, 2007.

[15] F. Sesti, O. E. Tsitsilonis, A. Kotsinas, and I. P. Trougakos, "Oxidative stress-mediated biomolecular damage and inflammation in tumorigenesis," In Vivo, vol. 26, no. 3, pp. 395-402, 2012.

[16] A. C. Schinzel and W. C. Hahn, "Oncogenic transformation and experimental models of human cancer," Frontiers in Bioscience, vol. 13, no. 1, pp. 71-84, 2008.

[17] N. O’Neil and A. Rose, "DNA repair," WormBook, vol. 13, pp. $1-12,2006$.

[18] B. Bilanges and D. Stokoe, "Mechanisms of translational deregulation in human tumors and therapeutic intervention strategies," Oncogene, vol. 26, no. 41, pp. 5973-5990, 2007.

[19] W. A. Freed-Pastor and C. Prives, "Mutant p53: one name, many proteins," Genes \& Development, vol. 26, no. 12, pp. 1268-1286, 2012.

[20] Y. Y. Liu, "Resuscitating wild-type p53 expression by disrupting ceramide glycosylation: a novel approach to target mutant p53 tumors," Cancer Research, vol. 71, no. 20, pp. 6295-6299, 2011.

[21] A. M. Goh, C. R. Coffill, and D. P. Lane, "The role of mutant p53 in human cancer," Journal of Pathology, vol. 223, no. 2, pp. 116-126, 2011.

[22] A. M. Bode and Z. Dong, "Post-translational modification of p53 in tumorigenesis," Nature Reviews Cancer, vol. 4, no. 10, pp. 793-805, 2004.
[23] X. Wang, "p53 regulation: teamwork between RING domains of Mdm2 and MdmX," Cell Cycle, vol. 10, no. 24, pp. 42254229, 2011.

[24] M. Hatakeyama and R. A. Weinberg, "The role of RB in cell cycle control," Progress in Cell Cycle Research, vol. 1, pp. 9-19, 1995.

[25] P. D. Adams, "Regulation of the retinoblastoma tumor suppressor protein by cyclin/cdks," Biochimica et Biophysica Acta, vol. 1471, no. 3, pp. M123-M133, 2001.

[26] G. Altavilla, A. Staffieri, G. Busatto, A. Canesso, L. Giacomelli, and G. Marioni, "Expression of p53, p16INK4A, pRb, p21WAF1/CIP1, p27KIP1, cyclin D1, Ki-67 and HPV DNA in sinonasal endophytic Schneiderian (inverted) papilloma," Acta Oto-Laryngologica, vol. 129, no. 11, pp. 1242-1249, 2009.

[27] M. J. Clemens, "Targets and mechanisms for the regulation of translation in malignant transformation," Oncogene, vol. 23, no. 18, pp. 3180-3188, 2004.

[28] J. Ren, B. N. Singh, Q. Huang et al., "DNA hypermethylation as a chemotherapy target," Cellular Signalling, vol. 23, no. 7, pp. 1082-1093, 2011.

[29] X. L. Hu, Y. Wang, and Q. Shen, "Epigenetic control on cell fate choice in neural stem cells," Protein \& Cell, vol. 3, no. 4, pp. 278-290, 2012.

[30] B. T. Spike and K. F. Macleod, "The Rb tumor suppressor in stress responses and hematopoietic homeostasis," Cell Cycle, vol. 4, no. 1, pp. 42-45, 2005.

[31] S. V. von Zeidler, E. C. Miracca, M. A. Nagai, and E. G. Birman, "Hypermethylation of the p16 gene in normal oral mucosa of smokers," International Journal of Molecular Medicine, vol. 14, no. 5, pp. 807-811, 2004.

[32] Y. Lu, B. Y. Zhang, Z. X. Jia, W. J. Wu, and Z. Q. Lu, "Hepatocellular carcinoma HepG2 cell apoptosis and caspase8 and Bcl-2 expression induced by injectable seed extract of coix lacryma-jobi," Hepatobiliary and Pancreatic Diseases International, vol. 10, no. 3, pp. 303-307, 2011.

[33] J. Gao, W. A. Morgan, A. Sanchez-Medina, and O. Corcoran, "The ethanol extract of Scutellaria baicalensis and the active compounds induce cell cycle arrest and apoptosis including upregulation of p53 and Bax in human lung cancer cells," Toxicology and Applied Pharmacology, vol. 254, no. 3, pp. 221228, 2011.

[34] S. J. Lee, K. Park, S. D. Ha, W. J. Kim, and S. K. Moon, "Gleditsia sinensis thorn extract inhibits human colon cancer cells: the role of ERK1/2, G2/M-phase cell cycle arrest and p53 expression," Phytotherapy Research, vol. 24, no. 12, pp. 18701876, 2010.

[35] Y. Lu, C. S. Li, and Q. Dong, “Chinese herb related molecules of cancer-cell-apoptosis: a minireview of progress between Kanglaite injection and related genes," Journal of Experimental and Clinical Cancer Research, vol. 27, no. 1, p. 31, 2008.

[36] B. Li, J. Zhao, C. Z. Wang et al., "Ginsenoside Rh2 induces apoptosis and paraptosis-like cell death in colorectal cancer cells through activation of p53," Cancer Letters, vol. 301, no. 2, pp. 185-192, 2011.

[37] H. Gali-Muhtasib, M. Diab-Assaf, C. Boltze et al., "Thymoquinone extracted from black seed triggers apoptotic cell death in human colorectal cancer cells via a p53-dependent mechanism," International Journal of Oncology, vol. 25, no. 4, pp. 857-866, 2004.

[38] E. R. Hahm and S. V. Singh, "Honokiol causes G0-G1 phase cell cycle arrest in human prostate cancer cells 
in association with suppression of retinoblastoma protein level/phosphorylation and inhibition of E2F1 transcriptional activity," Molecular Cancer Therapeutics, vol. 6, no. 10, pp. 2686-2695, 2007.

[39] R. Tao, L. Lu, R. Zhang, J. Hu, J. Ni, and W. Shen, "Triptolide inhibits rat vascular smooth muscle cell proliferation and cell cycle progression via attenuation of ERK $1 / 2$ and $\mathrm{Rb}$ phosphorylation," Experimental and Molecular Pathology, vol. 90, no. 2, pp. 137-142, 2011.

[40] B. E. Shan, K. Zeki, T. Sugiura, Y. Yoshida, and U. Yamashita, "Chinese medicinal herb, Acanthopanax gracilistylus, extract induces cell cycle arrest of human tumor cells in vitro," Japanese Journal of Cancer Research, vol. 91, no. 4, pp. 383$389,2000$.

[41] Y. Fu, T. C. Hsieh, J. Guo et al., "Licochalcone-A, a novel flavonoid isolated from licorice root (Glycyrrhiza glabra), causes G2 and late-G1 arrests in androgen-independent PC3 prostate cancer cells," Biochemical and Biophysical Research Communications, vol. 322, no. 1, pp. 263-270, 2004.

[42] X. Y. Xiao, M. Hao, X. Y. Yang et al., "Licochalcone A inhibits growth of gastric cancer cells by arresting cell cycle progression and inducing apoptosis," Cancer Letters, vol. 302, no. 1, pp. 69$75,2011$.

[43] S. Kametani, T. Oikawa, A. Kojima-Yuasa et al., "Mechanism of growth inhibitory effect of cape aloe extract in Ehrlich ascites tumor cells," Journal of Nutritional Science and Vitaminology, vol. 53, no. 6, pp. 540-546, 2007.

[44] P. Yang, C. Cartwright, D. Chan, M. Vijjeswarapu, J. Ding, and R. A. Newman, "Zyflamend $\AA$-mediated inhibition of human prostate cancer PC3 cell proliferation: effects on 12-LOX and Rb protein phosphorylation," Cancer Biology and Therapy, vol. 6, no. 2, pp. 228-236, 2007.

[45] H. Liu, C. Zang, A. Emde et al., "Anti-tumor effect of honokiol alone and in combination with other anti-cancer agents in breast cancer," European Journal of Pharmacology, vol. 591, no. 1-3, pp. 43-51, 2008.

[46] J. Y. Yang, M. A. Della-Fera, S. Rayalam, and C. A. Baile, "Enhanced effects of xanthohumol plus honokiol on apoptosis in 3T3-L1 adipocytes," Obesity, vol. 16, no. 6, pp. 1232-1238, 2008.

[47] S. Shankar and R. K. Srivastava, "Involvement of Bcl-2 family members, phosphatidylinositol 3'-kinase/AKT and mitochondrial p53 in curcumin (diferulolylmethane)- induced apoptosis in prostate cancer," International Journal of Oncology, vol. 30, no. 4, pp. 905-918, 2007.

[48] N. K. Narayanan, D. Nargi, C. Randolph, and B. A. Narayanan, "Liposome encapsulation of curcumin and resveratrol in combination reduces prostate cancer incidence in PTEN knockout mice," International Journal of Cancer, vol. 125, no. 1, pp. 1-8, 2009.

[49] H. Yoshida, N. Okumura, Y. Kitagishi, Y. Nishimura, and S. Matsuda, "Ethanol extract of Rosemary repressed PTEN expression in K562 culture cells," International Journal of Applied Biology and Pharmaceutical Technology, vol. 2, pp. 316-322, 2011.

[50] S. J. Lee, H. M. Kim, Y. H. Cho et al., "Aqueous extract of Magnolia officinalis mediates proliferative capacity, p21WAF1 expression and TNF-alpha-induced NF-kappaB activity in human urinary bladder cancer 5637 cells; involvement of p38 MAP kinase," Oncology Reports, vol. 18, no. 3, pp. 729-736, 2007.
[51] L. H. Dong, J. K. Wen, S. B. Miao et al., "Baicalin inhibits PDGF-BB-stimulated vascular smooth muscle cell proliferation through suppressing PDGFR $\beta$-ERK signaling and increase in p27 accumulation and prevents injury-induced neointimal hyperplasia," Cell Research, vol. 20, no. 11, pp. 1252-1262, 2010.

[52] S. J. Lee, S. S. Park, W. J. Kim, and S. K. Moon, "Gleditsia sinensis thorn extract inhibits proliferation and TNF- $\alpha$ induced MMP-9 expression in vascular smooth muscle cells," American Journal of Chinese Medicine, vol. 40, no. 2, pp. 373386, 2012.

[53] T. D. Way, J. C. Lee, D. H. Kuo et al., "Inhibition of epidermal growth factor receptor signaling by saussurea involucrata, a rare traditional chinese medicinal herb, in human hormoneresistant prostate cancer PC-3 cells," Journal of Agricultural and Food Chemistry, vol. 58, no. 6, pp. 3356-3365, 2010.

[54] Y. Huang, H. Song, H. Hu, L. Cui, C. You, and L. Huang, "Trichosanthin inhibits DNA methyltransferase and restores methylation-silenced gene expression in human cervical cancer cells," Molecular Medicine Reports, vol. 6, no. 4, pp. 872878, 2012.

[55] A. E. Moran, A. M. Carothers, M. J. Weyant, M. Redston, and M. M. Bertagnolli, "Carnosol inhibits $\beta$-catenin tyrosine phosphorylation and prevents adenoma formation in the C57BL/6J/Min/+ (Min/+) mouse," Cancer Research, vol. 65, no. 3, pp. 1097-1104, 2005. 


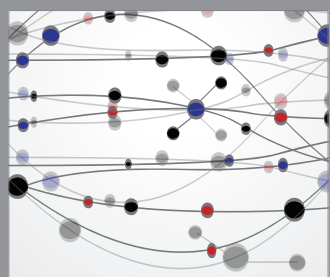

The Scientific World Journal
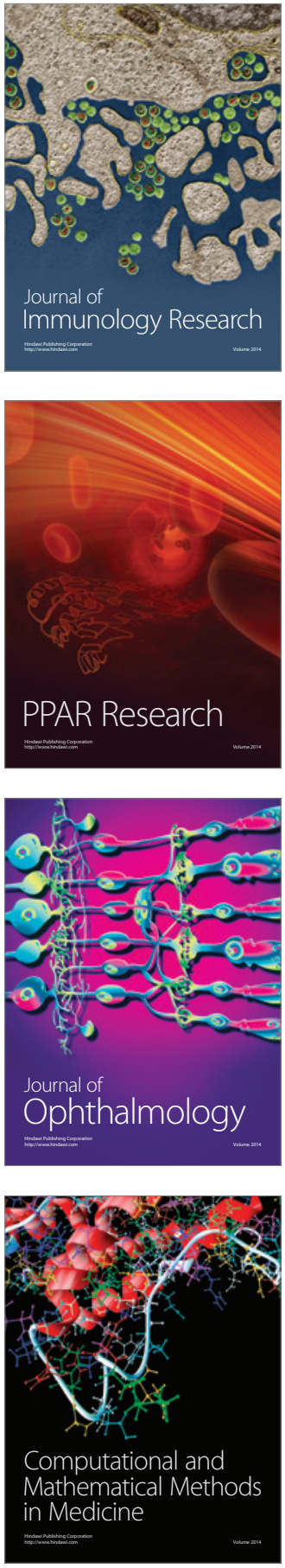

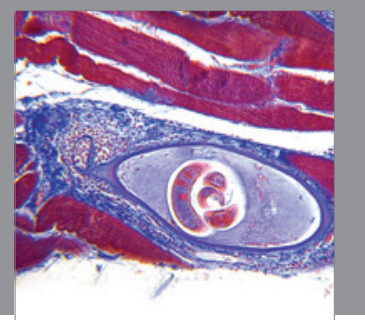

Gastroenterology

Research and Practice
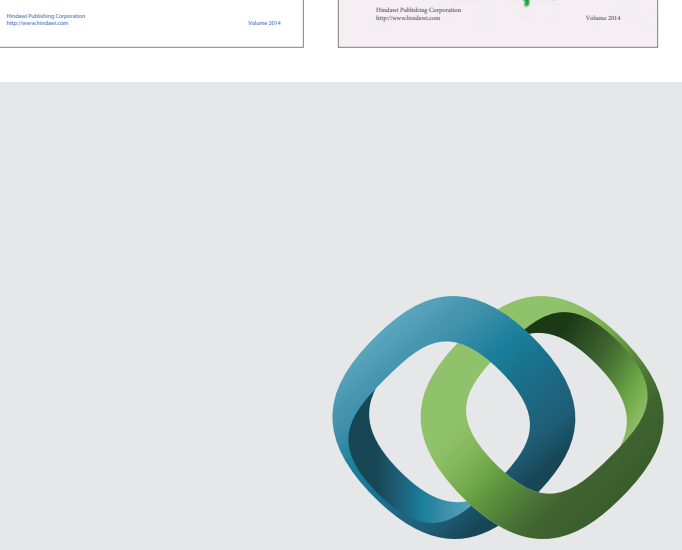

\section{Hindawi}

Submit your manuscripts at

http://www.hindawi.com
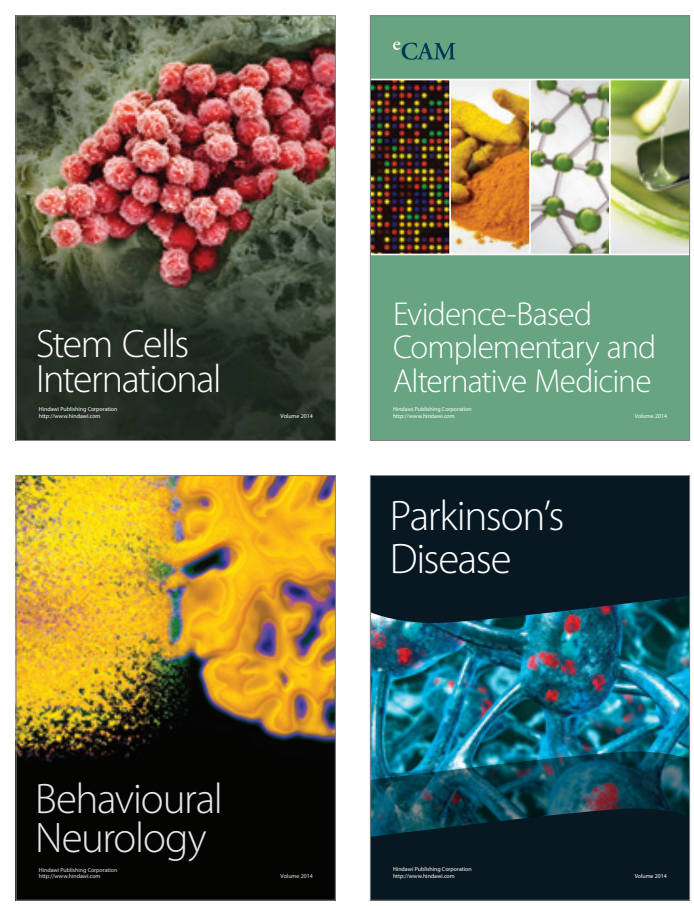

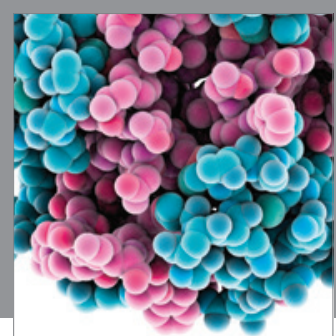

Journal of
Diabetes Research

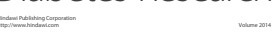

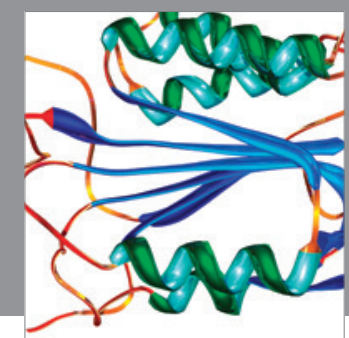

Disease Markers
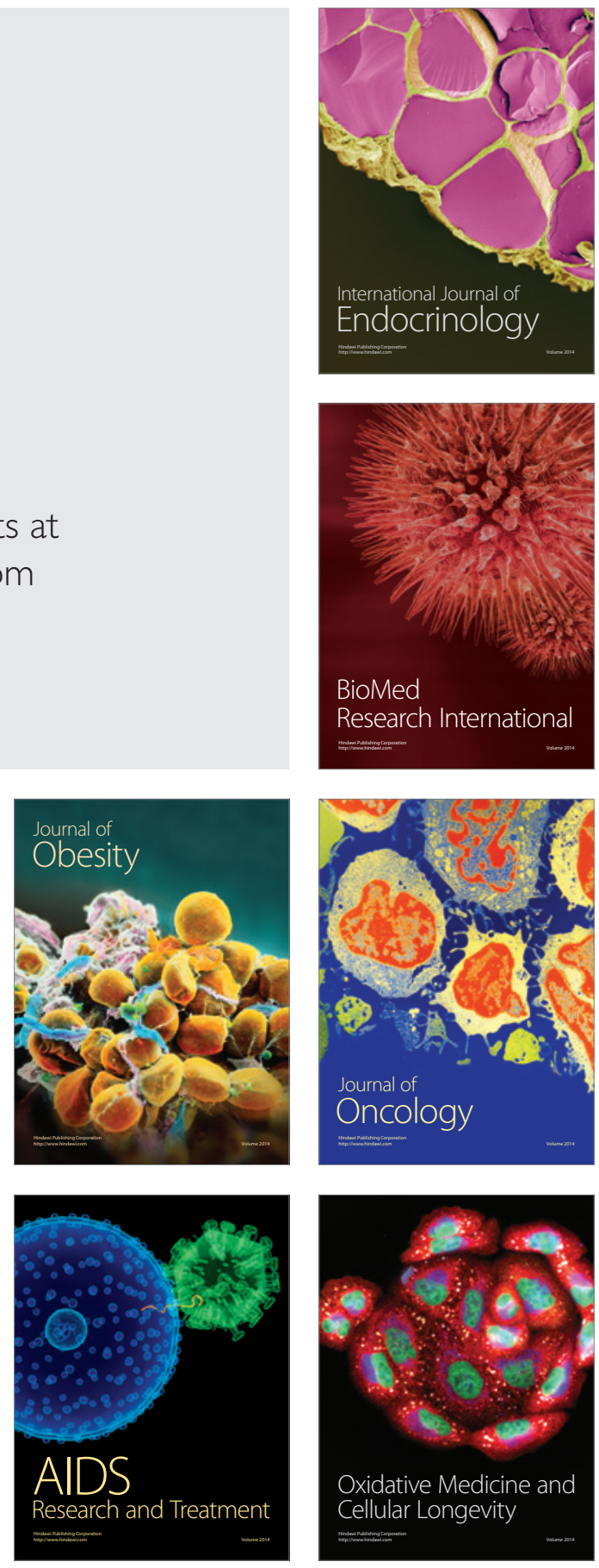